Mathematical Modelling and Analysis

Volume 17 Number 4, September 2012, 558-570

http://dx.doi.org/10.3846/13926292.2012.708675

(C) Vilnius Gediminas Technical University, 2012
Publisher: Taylor\&Francis and VGTU

http://www.tandfonline.com/TMMA

Print ISSN: 1392-6292

Online ISSN: 1648-3510

\title{
Numerical Analysis of an Implicit Fully Discrete Local Discontinuous Galerkin Method for the Fractional Zakharov-Kuznetsov Equation
}

\author{
Zongxiu Ren ${ }^{a}$, Leilei Wei ${ }^{b}$, Yinnian $\mathrm{He}^{b}$ and Shaoli Wang ${ }^{b}$ \\ ${ }^{a}$ Henan Normal University \\ 453007 Xinxiang, China \\ ${ }^{b}$ Xi'an Jiaotong University \\ 710049 Xi'an, China \\ E-mail: leileiwei09@gmail.com
}

Received October 29, 2011; revised June 28, 2012; published online September 1, 2012

\begin{abstract}
In this paper we develop and analyze an implicit fully discrete local discontinuous Galerkin (LDG) finite element method for a time-fractional ZakharovKuznetsov equation. The method is based on a finite difference scheme in time and local discontinuous Galerkin methods in space. We show that our scheme is unconditional stable and $L^{2}$ error estimate for the linear case with the convergence rate $O\left(h^{k+1}+(\Delta t)^{2}+(\Delta t)^{\frac{\alpha}{2}} h^{k+\frac{1}{2}}\right)$ through analysis.
\end{abstract}

Keywords: time-fractional partial differential equations, Zakharov-Kuznetsov equation, local discontinuous Galerkin method, stability, error estimates.

AMS Subject Classification: 35Q53; 65M60.

\section{Introduction}

During the last few decades the numerical modeling and simulation for fractional calculus have been the focus of many studies, and various fractional order differential equation have been solved including e.g. space-time fractional partial differential equation [10], space and time fractional Fokker-Planck equation [5], fractional order two point boundary value problem [7], the fractional $\mathrm{KdV}$ equation [13], fractional convection-diffusion equation [14], fractional partial differential equations fluid mechanics [15], fractional KdV-Burgers-Kuramoto equation [16] and so on. Solving such fractional partial differential using numerical schemes has been stimulated due to their frequent appearance in various applications in physics and engineering.

The Zakharov-Kuznetsov (ZK) equation is a generalization of the Korteweg-de Vries (KdV) equation. It was obtained by Zakharov and Kuznetsov [19] to describe the behavior of weakly nonlinear ion-acoustic waves in a plasma comprising cold ions and hot isothermal electrons in the presence of a uniform 
magnetic field. Several properties of this equation including existence and stability of solitary wave solutions have been extensively studied in the literature $[2,6]$. Some methods $[1,17]$ have been used to handle the integer-order systems, however, to the best of our knowledge, the study of the fractional ZakharovKuznetsov (ZK) equation has not been widespread.

In this paper, we consider the following time-fractional ZK equation

$$
\begin{gathered}
\frac{\partial^{\alpha} u(x, y, t)}{\partial t^{\alpha}}+g(u)_{x}+\left(\nabla^{2} u\right)_{x}=0, \quad(x, y, t) \in \Omega \times[0, T] \\
u(x, y, 0)=u_{0}(x, y), \quad x \in \Omega,
\end{gathered}
$$

where $\nabla^{2}=\partial_{x}^{2}+\partial_{y}^{2}$ is the isotropic Laplacian. $0<\alpha \leq 1$ is a parameter describing the order of the fractional time. In this paper we do not pay attention to boundary conditions, hence the solution is considered to be either periodic or compactly supported.

The time fractional derivative in the equation (1.1), uses the Caputo fractional partial derivative of order $\alpha$, defined as [11]

$$
\frac{\partial^{\alpha} u(x, y, t)}{\partial t^{\alpha}}= \begin{cases}\frac{1}{\Gamma(1-\alpha)} \int_{0}^{t} \frac{\partial u(x, y, s)}{\partial s} \frac{d s}{(t-s)^{\alpha}} & \text { if } 0<\alpha<1 \\ \frac{\partial u(x, y, t)}{\partial t} & \text { if } \alpha=1\end{cases}
$$

here $\Gamma(\cdot)$ is the Gamma function.

There are only a few numerical works in the literature to solve the fractional ZK equation. Molliq et al. [12] presents the approximate analytical solution of a fractional ZK equation using the variational iteration method. Yildirim [18] extended He's homotopy perturbation method to derive explicit and numerical solutions of fractional ZK equations. The discontinuous Galerkin finite element method is a very attractive method for partial differential equations because of its flexibility and efficiency in terms of mesh and shape functions, and the higher order of convergence can be achieved without over many iterations.

In the present paper we propose an implicit fully discrete local discontinuous Galerkin (LDG) finite element method for solving time-fractional ZK equation. Our fully discrete scheme is based on a finite difference scheme in time and local discontinuous Galerkin methods in space. Stability is ensured by a careful choice of interface numerical fluxes. We prove that our scheme is unconditionally stable and $L^{2}$ error estimate for the linear case with the convergence rate $O\left(h^{k+1}+(\Delta t)^{2}+(\Delta t)^{\alpha / 2} h^{k+\frac{1}{2}}\right)$.

This paper is organized as follows. First we introduce some basic notations and mathematical preliminaries, then in Section 3 we discuss the fully discrete LDG scheme for the fractional ZK equation (1.1), and prove that the scheme is unconditionally stable, and the numerical solution is convergent. Finally in Section 4 concluding remarks are provided. 


\section{Notations and Auxiliary Results}

\subsection{Notations and projection}

In the domain $[a, b] \times[c, d]$ we define cells

$$
I_{i, j}=\left\{(x, y): x_{i-\frac{1}{2}} \leq x \leq x_{i+\frac{1}{2}}, y_{j-\frac{1}{2}} \leq y \leq y_{j+\frac{1}{2}}\right\},
$$

for $i=1, \ldots, N_{x}, j=1, \ldots, N_{y}$, where

$$
a=x_{\frac{1}{2}}<x_{\frac{3}{2}}<\cdots<x_{N_{x}+\frac{1}{2}}=b, \quad c=y_{\frac{1}{2}}<y_{\frac{3}{2}}<\cdots<y_{N_{y}+\frac{1}{2}}=d,
$$

We define $I_{i}$ and $J_{j}$ as: $I_{i}=\left[x_{i-\frac{1}{2}}, x_{i+\frac{1}{2}}\right], J_{j}=\left[y_{j-\frac{1}{2}}, y_{j+\frac{1}{2}}\right]$ for $i=1, \ldots, N_{x}$, $j=1, \ldots, N_{y}$. So we have $I_{i, j}=I_{i}^{2} \times J_{j}$.

We denote by $u_{i+\frac{1}{2}, y}^{+}$and $u_{i+\frac{1}{2}, y}^{-}$the values of $u$ at $x_{i+1 / 2}$, from the right cell $I_{i+1} \times J_{j}$ and from the left cell $I_{i} \times J_{j}$ when $y \in J_{j}$, on all vertical edges, respectively. Similarly, we denote by $u_{x, j+1 / 2}^{+}$and $u_{x, j+1 / 2}^{-}$the values of $u$ at $y_{j+1 / 2}$, from the top cell $I_{i} \times J_{j+1}$ and from the bottom cell $I_{i} \times J_{j}$, when $x \in I_{i}$, on all horizontal edges, respectively.

Define the space $V_{h}^{k}$ as the space of tensor product piecewise polynomials of degree at most $k$ in each variable on every element, i.e.

$$
V_{h}^{k}=\left\{v: v \in Q^{k}\left(I_{i} \times J_{j}\right), \forall(x, y) \in I_{i} \times J_{j}, i=1, \ldots, N_{x}, j=1, \ldots, N_{y}\right\} .
$$

\subsection{Projection}

In order to prove the error estimates for two-dimensional problems in Cartesian meshes, we use the same projections as in [3]. However, for the sake of completeness, we present the definition. First, we will give the projection in one dimension $[a, b]$, denoted by $\mathcal{P}_{x}$, i.e.,for each $j$,

$$
\int_{I_{i}}\left(\mathcal{P}_{x} \omega(x)-\omega(x)\right) v(x)=0, \quad \forall v \in P^{k}\left(I_{i}\right),
$$

and special projection $\mathcal{P}_{x}^{ \pm}$, i.e., for each $j$,

$$
\begin{array}{ll}
\int_{I_{i}}\left(\mathcal{P}_{x}^{+} \omega(x)-\omega(x)\right) v(x)=0, \forall v \in P^{k-1}\left(I_{i}\right), & \mathcal{P}_{x}^{+} \omega\left(x_{i-\frac{1}{2}}^{+}\right)=\omega\left(x_{i-\frac{1}{2}}\right), \\
\int_{I_{i}}\left(\mathcal{P}_{x}^{-} \omega(x)-\omega(x)\right) v(x)=0, \forall v \in P^{k-1}\left(I_{i}\right), & \mathcal{P}_{x}^{-} \omega\left(x_{i+\frac{1}{2}}^{-}\right)=\omega\left(x_{i+\frac{1}{2}}\right) .
\end{array}
$$

Similarly, we can also define the projection $\mathcal{P}_{y}, \mathcal{P}_{y}^{+}, \mathcal{P}_{y}^{-}$.

On a rectangle $[a, b] \times[c, d]$, for a function $\omega(x, y)$, define

$$
\mathbb{P} \omega=\mathcal{P}_{x} \otimes \mathcal{P}_{y} \omega(x, y), \quad \mathbb{P}^{ \pm} \omega=\mathcal{P}_{x}^{ \pm} \otimes \mathcal{P}_{y}^{ \pm} \omega(x, y),
$$

where the subscripts indicate the application of the one dimensional operators $\mathcal{P}$ or $\mathcal{P}^{ \pm}$with respect to the corresponding variable. We list some properties for the projections $\mathbb{P}$ :

$$
\int_{I_{i}} \int_{J_{j}}\left(\mathbb{P}^{ \pm} \omega(x, y)-\omega(x, y)\right) v(x, y) d y d x=0,
$$


for any $v(x, y) \in\left(P^{k-1}\left(I_{i}\right) \otimes P^{k}\left(J_{j}\right)\right) \cup\left(P^{k}\left(I_{i}\right) \otimes P^{k-1}\left(J_{j}\right)\right)$. Also

$$
\begin{array}{ll}
\int_{I_{i}}\left(\mathbb{P}^{+} \omega\left(x, y_{j-\frac{1}{2}}^{+}\right)-\omega\left(x, y_{j-\frac{1}{2}}\right)\right) v\left(x, y_{j-\frac{1}{2}}^{+}\right)=0, & \forall v \in Q^{k}\left(I_{i} \otimes J_{j}\right), \\
\int_{I_{i}}\left(\mathbb{P}^{-} \omega\left(x, y_{j+\frac{1}{2}}^{-}\right)-\omega\left(x, y_{j+\frac{1}{2}}\right)\right) v\left(x, y_{j+\frac{1}{2}}^{-}\right)=0, & \forall v \in Q^{k}\left(I_{i} \otimes J_{j}\right)
\end{array}
$$

and

$$
\begin{array}{ll}
\int_{J_{j}}\left(\mathbb{P}^{+} \omega\left(x_{i-\frac{1}{2}}^{+}, y\right)-\omega\left(x_{i-\frac{1}{2}}, y\right)\right) v\left(x_{i-\frac{1}{2}}^{+}, y\right)=0, & \forall v \in Q^{k}\left(I_{i} \otimes J_{j}\right), \\
\int_{J_{j}}\left(\mathbb{P}^{-} \omega\left(x_{i+\frac{1}{2}}^{-}, y\right)-\omega\left(x_{i+\frac{1}{2}}, y\right)\right) v\left(x_{i+\frac{1}{2}}^{-}, y\right)=0, & \forall v \in Q^{k}\left(I_{i} \otimes J_{j}\right) .
\end{array}
$$

For the projections $(2.1)$, the following inequality holds $[3,8]$

$$
\left\|\omega^{e}\right\|+h\left\|\omega^{e}\right\|_{\infty}+h^{\frac{1}{2}}\left\|\omega^{e}\right\|_{\tau_{h}} \leq C h^{k+1}
$$

where $\omega^{e}=\mathbb{P} \omega-\omega$ or $\omega^{e}=\mathbb{P}^{ \pm} \omega-\omega$. The positive constant $C$, solely depending on $\omega$, is independent of $h$. $\left\|\omega^{e}\right\|$ and $\left\|\omega^{e}\right\|_{\tau_{h}}$ denote the $L^{2}$-norm of $\omega^{e}$ on $\Omega$ and $\tau_{h}$, which are

$$
\begin{aligned}
\left\|\omega^{e}\right\|=\quad & \left(\sum_{1 \leq i \leq N_{x}} \sum_{0 \leq j \leq N_{y}} \int_{I_{i}} \int_{J_{j}}\left(\omega^{e}\right)^{2}\right)^{\frac{1}{2}} \\
\left\|\omega^{e}\right\|_{\tau_{h}}=\quad & \left(\sum_{0 \leq i \leq N_{x}} \int_{c}^{d}\left(\left(\left(\omega^{e}\right)_{i+\frac{1}{2}, y}^{+}\right)^{2}+\left(\left(\omega^{e}\right)_{i+\frac{1}{2}, y}^{-}\right)^{2}\right) d y\right. \\
& \left.+\sum_{0 \leq j \leq N_{y}} \int_{a}^{b}\left(\left(\left(\omega^{e}\right)_{x, j+\frac{1}{2}}^{+}\right)^{2}+\left(\left(\omega^{e}\right)_{x, j+\frac{1}{2}}^{-}\right)^{2}\right) d x\right)^{\frac{1}{2}}
\end{aligned}
$$

respectively, and $\left\|\omega^{e}\right\|_{\infty}=\operatorname{ess} \sup \left|\omega^{e}\right|$. Here and below we use $C$ to denote a positive constant which may have a different value in each occurrence.

\section{$2.3 \quad$ Numerical flux}

In this paper we will be using the flux $\widehat{g}\left(\phi^{-}, \phi^{+}\right)$which is related to the discontinuous Galerkin spatial discretization. $\widehat{g}\left(\phi^{-}, \phi^{+}\right)$is a monotone numerical flux, which is dependent on the two values of the function $\phi$ at the discontinuity point $x_{j+\frac{1}{2}}$, and satisfies the following conditions:

(i) it is locally Lipschitz continuous, so it is bounded when $\phi^{ \pm}$are bounded;

(ii) it is consistent with the flux $g(\phi)$, i.e., $\widehat{g}(\phi, \phi)=g(\phi)$;

(iii) it is a nondecreasing function of its first argument, and a nonincreasing function of its second argument. 


\section{Fully Discrete LDG Scheme}

In this section we introduce the numerical scheme for the solution of equation (1.1). Let $\Delta t=T / M$ be the time mesh size, $M$ is a positive integer, $t_{n}=n \Delta t, n=0,1, \ldots, M$ be mesh point. An approximation to time fractional derivative (1.2) can be obtained by simple quadrature formula given as [9]

$$
\frac{\partial^{\alpha} u\left(x, y, t_{n}\right)}{\partial t^{\alpha}}=\frac{(\Delta t)^{1-\alpha}}{\Gamma(2-\alpha)} \sum_{m=0}^{n-1} b_{m} \frac{u\left(x, y, t_{n-m}\right)-u\left(x, y, t_{n-m-1}\right)}{\Delta t}+\gamma^{n}(x, y),
$$

where $b_{m}=(m+1)^{1-\alpha}-m^{1-\alpha}, \gamma^{n}(x, y) \leq C(\Delta t)^{2-\alpha}, C$ is dependent on $u$, $T, \alpha$. We know that

$$
\begin{aligned}
& 1=b_{0}>b_{1}>b_{2}>\cdots>b_{n}>0, \quad b_{n} \rightarrow 0(n \rightarrow \infty), \\
& \sum_{m=1}^{n}\left(b_{m-1}-b_{m}\right)+b_{n}=1 .
\end{aligned}
$$

We rewrite Eq. (1.1) as a system:

$$
\begin{aligned}
& p=u_{x}, \quad q=p_{x}, \quad s=u_{y}, \quad r=s_{x}, \\
& \frac{\partial^{\alpha} u(x, y, t)}{\partial t^{\alpha}}+g(u)_{x}+q_{x}+r_{y}=0 .
\end{aligned}
$$

Let $u_{h}^{n}, p_{h}^{n}, q_{h}^{n}, r_{h}^{n}, s_{h}^{n} \in V_{h}^{k}$ be the approximations of $u\left(\cdot, t_{n}\right), p\left(\cdot, t_{n}\right), q\left(\cdot, t_{n}\right)$, $r\left(\cdot, t_{n}\right), s\left(\cdot, t_{n}\right)$, respectively, $f^{n}(x)=f\left(x, t_{n}\right)$. With $(3.1)$, we define a fully discrete local discontinuous Galerkin scheme as follows: find $u_{h}^{n}, p_{h}^{n}, q_{h}^{n}, r_{h}^{n}, s_{h}^{n} \in$ $V_{h}^{k}$, such that for all test functions $v, \rho, \xi, \eta, \phi \in V_{h}^{k}$,

$$
\begin{gathered}
\int_{a}^{b} \int_{c}^{d} u_{h}^{n} v d x d y-\beta\left(\int_{a} \int_{c}^{b} g\left(u_{h}^{n}\right) v_{x} d x d y-\sum_{i=1}^{N_{x}} \int_{c}^{d}\left(\left(\widehat{g} v^{-}\right)_{i+\frac{1}{2}, y}-\left(\widehat{g} v^{+}\right)_{i-\frac{1}{2}, y}\right) d y\right) \\
-\beta\left(\int_{a}^{b} \int_{c}^{d} q_{h}^{n} v_{x} d x d y-\sum_{i=1}^{N_{x}} \int_{c}^{d}\left(\left(\widehat{q_{h}^{n}} v^{-}\right)_{i+\frac{1}{2}, y}-\left(\widehat{q_{h}^{n}} v^{+}\right)_{i-\frac{1}{2}, y}\right) d y\right) \\
\quad-\beta\left(\int_{a}^{b} \int_{c}^{d} r_{h}^{n} v_{y} d x d y-\sum_{j=1}^{N_{y}} \int_{a}^{b}\left(\left(\widetilde{r_{h}^{n}} v^{-}\right)_{x, j+\frac{1}{2}}-\left(\widetilde{r_{h}^{n}} v^{+}\right)_{x, j-\frac{1}{2}, y}\right) d x\right) \\
=\sum_{m=1}^{n-1}\left(b_{m-1}-b_{m}\right) \int_{a}^{b} \int_{c}^{d} u_{h}^{n-m} v d x d y+b_{n-1} \int_{a}^{b} \int_{c}^{d} u_{h}^{0} v d x d y, \\
\int_{\Omega} q_{h}^{n} \rho d x d y+\int_{a}^{b} \int_{c}^{d} p_{h}^{n} \rho_{x} d x d y-\sum_{i=1}^{N_{x}} \int_{c}^{d}\left(\left(\widehat{p_{h}^{n}} \rho^{-}\right)_{i+\frac{1}{2}, y}-\left(\widehat{p_{h}^{n}} \rho^{+}\right)_{i-\frac{1}{2}, y}\right) d y=0, \\
\int_{\Omega} p_{h}^{n} \xi d x d y+\int_{a}^{b} \int_{c}^{d} u_{h}^{n} \xi_{x} d x d y-\sum_{i=1}^{N_{x}} \int_{c}^{d}\left(\left(\widehat{u_{h}^{n}} \xi^{-}\right)_{i+\frac{1}{2}, y}-\left(\widehat{u_{h}^{n}} \xi^{+}\right)_{i-\frac{1}{2}, y}\right) d y=0, \\
\int_{\Omega} r_{h}^{n} \eta d x d y+\int_{a}^{b} \int_{c}^{d} s_{h}^{n} \eta_{x} d x d y-\sum_{i=1}^{N_{x}} \int_{c}^{d}\left(\left(\widehat{s_{h}^{n}} \eta^{-}\right)_{i+\frac{1}{2}, y}-\left(\widehat{s_{h}^{n}} \eta^{+}\right)_{i-\frac{1}{2}, y}\right) d y=0,
\end{gathered}
$$


$\int_{\Omega} s_{h}^{n} \phi d x d y+\int_{a}^{b} \int_{c}^{d} u_{h}^{n} \phi_{y} d x d y-\sum_{j=1}^{N_{y}} \int_{a}^{b}\left(\left(\widetilde{u_{h}^{n}} \phi^{-}\right)_{x, j+\frac{1}{2}}-\left(\widetilde{u_{h}^{n}} \phi^{+}\right)_{x, j-\frac{1}{2}, y}\right) d x=0$

where $\beta=(\Delta t)^{\alpha} \Gamma(2-\alpha)$. From (3.1) we know the truncation error is $\gamma^{n}(x)$.

The "hat" terms in (3.3) in the cell boundary terms from integration by parts are "numerical fluxes", in order to ensure stability, we can take the following choices simply

$$
\begin{aligned}
& \widehat{u_{h}^{n}}=\left(u_{h}^{n}\right)^{-}, \quad \widehat{p_{h}^{n}}=\left(p_{h}^{n}\right)^{+}, \quad \widehat{q_{h}^{n}}=\left(q_{h}^{n}\right)^{+}, \quad \widetilde{u_{h}^{n}}=\left(u_{h}^{n}\right)^{-}, \\
& \widetilde{r_{h}^{n}}=\left(r_{h}^{n}\right)^{+}, \quad \widetilde{r_{h}^{n}}=\widetilde{r_{h}^{n}}-\tau\left[u_{h}^{n}\right], \quad \widehat{s_{h}^{n}}=\left(s_{h}^{n}\right)^{+},
\end{aligned}
$$

here $\tau>0$. In order to control the boundary terms, a dissipative term is added in the flux $\widetilde{r_{h}^{n}}$. The flux $\widehat{g}\left(\left(u_{h}^{n}\right)^{-},\left(u_{h}^{n}\right)^{+}\right)$is a monotone flux as described in (2.3). Examples of monotone fluxes which are suitable for the local discontinuous Galerkin methods can be found in, e.g., [4]. For example, one could use the Lax-Friedrichs flux, which is given by

$$
\widehat{g}^{L F}\left(w^{-}, w^{+}\right)=\frac{1}{2}\left(g\left(w^{-}\right)+g\left(w^{+}\right)-\lambda_{0}\left(w^{+}-w^{-}\right)\right), \quad \lambda_{0}=\max _{w}\left|g^{\prime}(w)\right|
$$

We remark that the choice for the fluxes (3.4) is not unique. In fact the crucial part is taking $\widehat{u_{h}^{n}}$ and $\widehat{p_{h}^{n}}$ from opposite sides, $\widetilde{u_{h}^{n}}$ and $\underset{r_{h}^{n}}{~ f r o m ~ o p p o s i t e ~}$ sides.

Since the problem is nonlinear, we can use an iterative method to solve the problem. Now the definition of the algorithm is complete.

Next we consider the numerical analysis of the scheme (3.3). First we examine the stability property.

Theorem 1. For periodic or compactly supported boundary conditions, fullydiscrete LDG scheme (3.3) with flux (3.4) is unconditionally stable, and the numerical solution $u_{h}^{n}$ satisfies

$$
\begin{aligned}
\left\|u_{h}^{n}\right\|^{2} & +\beta \sum_{i=1}^{N_{x}} \int_{c}^{d}\left(\left[p_{h}^{n}\right]^{2}+\left[s_{h}^{n}\right]^{2}\right)_{i-\frac{1}{2}, y} d y+2 \beta \tau \sum_{j=1}^{N_{y}} \int_{a}^{b}\left[u_{h}^{n}\right]_{x, j-\frac{1}{2}}^{2} d x \\
& \leq\left\|u_{h}^{0}\right\|^{2}, \quad n=0,1, \ldots, M
\end{aligned}
$$

Proof. Taking the test functions $v=u_{h}^{n}, \rho=\beta p_{h}^{n}, \xi=-\beta q_{h}^{n}, \eta=\beta s_{h}^{n}$, 
$\phi=-\beta r_{h}^{n}$ in scheme (3.3), and with the fluxes choice (3.4) we obtain

$$
\begin{aligned}
\left\|u_{h}^{n}\right\|^{2} & +\beta \widetilde{G}\left(u_{h}^{n}\right)+\frac{\beta}{2} \sum_{i=1}^{N_{x}} \int_{c}^{d}\left(\left[p_{h}^{n}\right]^{2}+\left[s_{h}^{n}\right]^{2}\right)_{i-\frac{1}{2}, y} d y+\beta \tau \sum_{j=1}^{N_{y}} \int_{a}^{b}\left[u_{h}^{n}\right]_{x, j-\frac{1}{2}}^{2} d x \\
& +\beta \sum_{i=1}^{N_{x}} \int_{c}^{d}\left(\Psi_{1}\left(u_{h}^{n}, q_{h}^{n}\right)_{i+\frac{1}{2}, y}-\Psi_{1}\left(u_{h}^{n}, q_{h}^{n}\right)_{i-\frac{1}{2}, y}+\Theta_{1}\left(u_{h}^{n}, q_{h}^{n}\right)_{i-\frac{1}{2}, y}\right) d y \\
& +\beta \sum_{j=1}^{N_{y}} \int_{a}^{b}\left(\Psi_{2}\left(u_{h}^{n}, r_{h}^{n}\right)_{x, j+\frac{1}{2}}-\Psi_{2}\left(u_{h}^{n}, r_{h}^{n}\right)_{x, j-\frac{1}{2}}+\Theta_{2}\left(u_{h}^{n}, r_{h}^{n}\right)_{x, j-\frac{1}{2}}\right) d x \\
= & \sum_{m=1}^{n-1}\left(b_{m-1}-b_{m}\right) \int_{a}^{b} \int_{c}^{d} u_{h}^{n-m} u_{h}^{n} d x d y+b_{n-1} \int_{a}^{b} \int_{c}^{d} u_{h}^{0} u_{h}^{n} d x d y,
\end{aligned}
$$

here

$$
\begin{gathered}
\widetilde{G}\left(u_{h}^{n}\right)=-\int_{a}^{b} \int_{c}^{d} g\left(u_{h}^{n}\right) v_{x} d x d y+\sum_{i=1}^{N_{x}} \int_{c}^{d}\left(\left(\widehat{g} v^{-}\right)_{i+\frac{1}{2}, y}-\left(\widehat{g} v^{+}\right)_{i-\frac{1}{2}, y}\right) d y \\
\Psi_{1}\left(u_{h}^{n}, q_{h}^{n}\right)=-\left(q_{h}^{n}\right)^{-}\left(u_{h}^{n}\right)^{-}+\widehat{q_{h}^{n}}\left(u_{h}^{n}\right)^{-}+\widehat{u_{h}^{n}}\left(q_{h}^{n}\right)^{-}, \\
\Psi_{2}\left(u_{h}^{n}, r_{h}^{n}\right)=-\left(r_{h}^{n}\right)^{-}\left(u_{h}^{n}\right)^{-}+\widehat{\widetilde{r}}\left(u_{h}^{n}\right)^{-}+\widetilde{u_{h}^{n}}\left(r_{h}^{n}\right)^{-}, \\
\Theta_{1}\left(u_{h}^{n}, q_{h}^{n}\right)=-\left(q_{h}^{n}\right)^{-}\left(u_{h}^{n}\right)^{-}+\left(q_{h}^{n}\right)^{+}\left(u_{h}^{n}\right)^{+}+\widehat{q_{h}^{n}}\left(u_{h}^{n}\right)^{-}-\widehat{q_{h}^{n}}\left(u_{h}^{n}\right)^{+} \\
\quad+\widehat{u_{h}^{n}}\left(q_{h}^{n}\right)^{-}-\widehat{u_{h}^{n}}\left(q_{h}^{n}\right)^{+}, \\
\Theta_{2}\left(u_{h}^{n}, r_{h}^{n}\right)=-\left(r_{h}^{n}\right)^{-}\left(u_{h}^{n}\right)^{-}+\left(r_{h}^{n}\right)^{+}\left(u_{h}^{n}\right)^{+}+\widetilde{\widetilde{r_{h}^{n}}}\left(u_{h}^{n}\right)^{-}-\widetilde{r_{h}^{n}}\left(u_{h}^{n}\right)^{+} \\
\quad+\widetilde{u_{h}^{n}}\left(r_{h}^{n}\right)^{-}-\widetilde{u_{h}^{n}}\left(r_{h}^{n}\right)^{+}
\end{gathered}
$$

If we take fluxes (3.4) then after some simple calculation, we easily obtain $\Theta_{1}\left(u_{h}^{n}, q_{h}^{n}\right)=\Theta_{2}\left(u_{h}^{n}, r_{h}^{n}\right)=0$. Let us denote $G(u)=\int_{0}^{u} g(u) d u$, and use a mean value theorem, then for the nonlinear term we obtain

$$
\widetilde{G}\left(u_{h}^{n}\right)=\sum_{i=1}^{N_{x}} \int_{c}^{d}\left(G^{\prime}(\eta)-\widehat{g}\right)\left[u_{h}^{n}\right]_{i-\frac{1}{2}, y} d y \geq 0
$$

where $\eta$ is a value between $\left(u_{h}^{n}\right)_{i-\frac{1}{2}, y}^{-}$and $\left(u_{h}^{n}\right)_{i-\frac{1}{2}, y}^{+}$. By the monotonicity of flux function $\widehat{g}$ we have the inequality (3.6).

Then based on the equation (3.5), and by the property (3.2), we can get

$$
\begin{array}{r}
\left\|u_{h}^{n}\right\|^{2}+\frac{\beta}{2} \sum_{i=1}^{N_{x}} \int_{c}^{d}\left(\left[p_{h}^{n}\right]^{2}+\left[s_{h}^{n}\right]^{2}\right)_{i-\frac{1}{2}, y} d y+\beta \tau \sum_{j=1}^{N_{y}} \int_{a}^{b}\left[u_{h}^{n}\right]_{x, j-\frac{1}{2}}^{2} d x \\
=\sum_{m=1}^{n-1}\left(b_{m-1}-b_{m}\right) \int_{a}^{b} \int_{c}^{d} u_{h}^{n-m} u_{h}^{n} d x d y+b_{n-1} \int_{a}^{b} \int_{c}^{d} u_{h}^{0} u_{h}^{n} d x d y
\end{array}
$$




$$
\begin{aligned}
& \leq \sum_{m=1}^{n-1}\left(b_{m-1}-b_{m}\right)\left\|u_{h}^{n-m}\right\|\left\|u_{h}^{n}\right\|+b_{n-1}\left\|u_{h}^{0}\right\|\left\|u_{h}^{n}\right\| \\
& \leq \frac{1}{2}\left(\sum_{m=1}^{n-1}\left(b_{m-1}-b_{m}\right)\left\|u_{h}^{n-m}\right\|^{2}+b_{n-1}\left\|u_{h}^{0}\right\|^{2}\right)+\frac{1}{2}\left\|u_{h}^{n}\right\|^{2},
\end{aligned}
$$

that is

$$
\begin{aligned}
& \left\|u_{h}^{n}\right\|^{2}+\beta \sum_{i=1}^{N_{x}} \int_{c}^{d}\left(\left[p_{h}^{n}\right]^{2}+\left[s_{h}^{n}\right]^{2}\right)_{i-\frac{1}{2}, y} d y+2 \beta \tau \sum_{j=1}^{N_{y}} \int_{a}^{b}\left[u_{h}^{n}\right]_{x, j-\frac{1}{2}}^{2} d x \\
& \leq \sum_{m=1}^{n-1}\left(b_{m-1}-b_{m}\right)\left\|u_{h}^{n-m}\right\|^{2}+b_{n-1}\left\|u_{h}^{0}\right\|^{2} .
\end{aligned}
$$

We will prove the Theorem 1 by mathematical induction. When $n=1$, and from the inequality (3.7), we have

$$
\left\|u_{h}^{1}\right\|^{2}+\beta \sum_{i=1}^{N_{x}} \int_{c}^{d}\left(\left[p_{h}^{1}\right]^{2}+\left[s_{h}^{1}\right]^{2}\right)_{i-\frac{1}{2}, y} d y+2 \beta \tau \sum_{j=1}^{N_{y}} \int_{a}^{b}\left[u_{h}^{1}\right]_{x, j-\frac{1}{2}}^{2} d x \leq\left\|u_{h}^{0}\right\|^{2} .
$$

and $\left\|u_{h}^{1}\right\| \leq\left\|u_{h}^{0}\right\|$. Now suppose the following inequalities hold $\left\|u_{h}^{m}\right\| \leq\left\|u_{h}^{0}\right\|$, $m=1,2, \ldots, K$. Let take $n=K+1$ in the inequality (3.7), then we obtain

$$
\begin{aligned}
& \left\|u_{h}^{K+1}\right\|^{2}+\beta \sum_{i=1}^{N_{x}} \int_{c}^{d}\left(\left[p_{h}^{K+1}\right]^{2}+\left[s_{h}^{K+1}\right]^{2}\right)_{i-\frac{1}{2}, y} d y+2 \beta \tau \sum_{j=1}^{N_{y}} \int_{a}^{b}\left[u_{h}^{K+1}\right]_{x, j-\frac{1}{2}}^{2} d x \\
& \quad \leq \sum_{m=1}^{K}\left(b_{m-1}-b_{m}\right)\left\|u_{h}^{K+1-k}\right\|^{2}+b_{K}\left\|u_{h}^{0}\right\|^{2} \\
& \quad \leq\left(\sum_{m=1}^{K}\left(b_{m-1}-b_{m}\right)+b_{K}\right)\left\|u_{h}^{0}\right\|^{2} \leq\left\|u_{h}^{0}\right\|^{2} .
\end{aligned}
$$

This finishes the proof of the stability result.

Next we will state the error estimate of the scheme for the linear case $g(u)=u$, and use (3.4) as our flux choice. We have the following theorem.

Theorem 2. Let $u\left(x, y, t_{n}\right)$ be the exact solution of the problem (1.1), $u_{h}^{n}$ be the numerical solution of the fully discrete LDG scheme (3.3), then there holds the following error estimates

$$
\left\|u\left(x, y, t_{n}\right)-u_{h}^{n}\right\| \leq C\left(h^{k+1}+(\Delta t)^{2}+(\Delta t)^{\frac{\alpha}{2}} h^{k+\frac{1}{2}}\right) .
$$

Proof. For convenience we define the notations

$$
\begin{aligned}
& \mathcal{R}(\omega, \widehat{\omega} ; \phi)=\int_{a}^{b} \int_{c}^{d} \omega \phi_{x} d x d y-\sum_{i=1}^{N_{x}} \int_{c}^{d}\left(\left(\widehat{\omega} \phi^{-}\right)_{i+\frac{1}{2}, y}-\left(\widehat{\omega} \phi^{+}\right)_{i-\frac{1}{2}, y}\right) d y \\
& \mathcal{Z}(\omega, \widetilde{\omega} ; \phi)=\int_{a}^{b} \int_{c}^{d} \omega \phi_{y} d x d y-\sum_{j=1}^{N_{y}} \int_{a}^{b}\left(\left(\widetilde{\omega} \phi^{-}\right)_{x, j+\frac{1}{2}}-\left(\widetilde{\omega} \phi^{+}\right)_{x, j-\frac{1}{2}}\right) d x .
\end{aligned}
$$


It is easy to verify that the exact solution of PDE (1.1) satisfies

$$
\begin{aligned}
& \int_{a}^{b} \int_{c}^{d} u\left(x, y, t_{n}\right) v d x d y+\beta \int_{a}^{b} \int_{c}^{d} \gamma^{n}(x, y) v d x d y-\beta \mathcal{R}\left(u\left(x, y, t_{n}\right), u\left(x, y, t_{n}\right) ; v\right) \\
& \quad-\beta \mathcal{R}\left(q\left(x, y, t_{n}\right), q\left(x, y, t_{n}\right) ; v\right)-\beta \mathcal{Z}\left(r\left(x, y, t_{n}\right), r\left(x, y, t_{n}\right) ; v\right) \\
& =\sum_{m=1}^{n-1}\left(b_{m-1}-b_{m}\right) \int_{a}^{b} \int_{c}^{d} u\left(x, y, t_{n-m}\right) v d x d y+b_{n-1} \int_{a}^{b} \int_{c}^{d} u\left(x, u, t_{0}\right) v d x d y, \\
& \int_{a}^{b} \int_{c}^{d} q\left(x, y, t_{n}\right) \rho d x d y+\mathcal{R}\left(p\left(x, y, t_{n}\right), p\left(x, y, t_{n}\right) ; \rho\right)=0 \\
& \int_{a}^{b} \int_{c}^{d} p\left(x, y, t_{n}\right) \xi d x d y+\mathcal{R}\left(u\left(x, y, t_{n}\right), u\left(x, y, t_{n}\right) ; \xi\right)=0 \\
& \int_{a}^{b} \int_{c}^{d} r\left(x, y, t_{n}\right) \eta d x d y+\mathcal{R}\left(s\left(x, y, t_{n}\right), s\left(x, y, t_{n}\right) ; \eta\right)=0 \\
& \int_{a}^{b} \int_{c}^{d} s\left(x, y, t_{n}\right) \phi d x d y+\mathcal{Z}\left(u\left(x, y, t_{n}\right), u\left(x, y, t_{n}\right) ; \phi\right)=0 .
\end{aligned}
$$

Denote

$$
\begin{aligned}
e_{u}^{n} & =u\left(x, y, t_{n}\right)-u_{h}^{n}=\mathcal{P}^{-} e_{u}^{n}-\left(\mathcal{P}^{-} u\left(x, y, t_{n}\right)-u\left(x, y, t_{n}\right)\right), \\
e_{q}^{n} & =q\left(x, y, t_{n}\right)-q_{h}^{n}=\mathcal{P} e_{q}^{n}-\left(\mathcal{P} q\left(x, y, t_{n}\right)-q\left(x, y, t_{n}\right)\right), \\
e_{p}^{n} & =p\left(x, y, t_{n}\right)-p_{h}^{n}=\mathcal{P} e_{p}^{n}-\left(\mathcal{P} p\left(x, y, t_{n}\right)-p\left(x, y, t_{n}\right)\right), \\
e_{r}^{n} & =r\left(x, y, t_{n}\right)-r_{h}^{n}=\mathcal{P} e_{r}^{n}-\left(\mathcal{P} r\left(x, y, t_{n}\right)-r\left(x, y, t_{n}\right)\right), \\
e_{s}^{n} & =s\left(x, y, t_{n}\right)-s_{h}^{n}=\mathcal{P} e_{s}^{n}-\left(\mathcal{P} s\left(x, y, t_{n}\right)-s\left(x, y, t_{n}\right)\right) .
\end{aligned}
$$

Subtracting (3.3) from (3.8), and with the fluxes (3.4) we can obtain the error equation

$$
\begin{aligned}
& \int_{a}^{b} \int_{c}^{d} e_{u}^{n} v d x d y-\beta \mathcal{R}\left(e_{u}^{n},\left(e_{u}^{n}\right)^{-} ; v\right)-\beta \mathcal{R}\left(e_{q}^{n},\left(e_{q}^{n}\right)^{+} ; v\right)-\beta \mathcal{Z}\left(e_{r}^{n},\left(e_{r}^{n}\right)^{+} ; v\right) \\
& \quad+\int_{\Omega} e_{q}^{n} \rho d x d y+\mathcal{R}\left(e_{p}^{n},\left(e_{p}^{n}\right)^{+} ; \rho\right)+\int_{\Omega} e_{p}^{n} \xi d x d y+\mathcal{R}\left(e_{u}^{n},\left(e_{u}^{n}\right)^{-} ; \xi\right) \\
& \quad+\int_{\Omega} e_{r}^{n} \eta d x d y+\mathcal{R}\left(e_{s}^{n},\left(e_{s}^{n}\right)^{+} ; \eta\right)+\int_{\Omega} e_{s}^{n} \phi d x d y+\mathcal{Z}\left(e_{u}^{n},\left(e_{u}^{n}\right)^{-} ; \phi\right) \\
& \quad-\sum_{m=1}^{n-1}\left(b_{m-1}-b_{m}\right) \int_{a}^{b} \int_{c}^{d} e_{u}^{n-m} v d x d y-b_{n-1} \int_{a}^{b} \int_{c}^{d} e_{u}^{0} v d x d y \\
& \quad+\beta \int_{a}^{b} \int_{c}^{d} \gamma^{n}(x, y) v d x+\beta \tau \sum_{j=1}^{N_{y}} \int_{a}^{b}\left[e_{u}^{n}\right]_{x, j-\frac{1}{2}}^{2} d x=0 .
\end{aligned}
$$

Taking $v=\mathcal{P}^{-} e_{u}^{n}, \rho=\beta \mathcal{P} e_{p}^{n}, \xi=-\beta \mathcal{P} e_{q}^{n}, \eta=\beta \mathcal{P} e_{s}^{n}, \phi=-\beta \mathcal{P} e_{r}^{n}$. 
Using (3.9), the properties (2.2) and (2.3) gives

$$
\begin{aligned}
& \int_{a}^{b} \int_{c}^{d}\left(\mathcal{P}^{-} e_{u}^{n}\right)^{2} d x+\beta \tau \sum_{j=1}^{N_{y}} \int_{a}^{b}\left[\mathcal{P}^{-} e_{u}^{n}\right]_{x, j-\frac{1}{2}}^{2} d x \\
& +\frac{\beta}{2} \sum_{i=1}^{N_{x}} \int_{c}^{d}\left(\left[\mathcal{P}^{-} e_{u}^{n}\right]^{2}+\left[\mathcal{P} e_{p}^{n}\right]^{2}+\left[\mathcal{P} e_{s}^{n}\right]^{2}\right)_{i-\frac{1}{2}, y} d y \\
& =\int_{a}^{b} \int_{c}^{d}\left(\mathcal{P}^{-} u\left(x, y, t_{n}\right)-u\left(x, y, t_{n}\right)\right) \mathcal{P}^{-} e_{u}^{n} d x d y-\beta \int_{a}^{b} \int_{c}^{d} \gamma^{n}(x, y) \mathcal{P}^{-} e_{u}^{n} d x d y \\
& -\beta \sum_{i=1}^{N_{x}} \int_{c}^{d}\left\{\left(\mathcal{P} q\left(x, y, t_{n}\right)-q\left(x, y, t_{n}\right)\right)^{+}\left[\mathcal{P}^{-} e_{u}^{n}\right]\right. \\
& -\left(\mathcal{P} s\left(x, y, t_{n}\right)-s\left(x, y, t_{n}\right)\right)^{+}\left[\mathcal{P} e_{s}^{n}\right] \\
& \left.-\left(\mathcal{P} p\left(x, y, t_{n}\right)-p\left(x, y, t_{n}\right)\right)^{+}\left[\mathcal{P} e_{p}^{n}\right]\right\}_{i-\frac{1}{2}, y} d y \\
& +\sum_{m=1}^{n-1}\left(b_{m-1}-b_{m}\right) \int_{a}^{b} \int_{c}^{d} \mathcal{P}^{-} e_{u}^{n-m} \mathcal{P}^{-} e_{u}^{n} d x d y+b_{n-1} \int_{a}^{b} \int_{c}^{d} \mathcal{P}^{-} e_{u}^{0} \mathcal{P}^{-} e_{u}^{n} d x d y \\
& -\sum_{m=1}^{n-1}\left(b_{m-1}-b_{m}\right) \int_{a}^{b} \int_{c}^{d}\left(\mathcal{P}^{-} u\left(x, y, t_{n-m}\right)-u\left(x, y, t_{n-m}\right)\right) \mathcal{P}^{-} e_{u}^{n} d x d y \\
& -\beta \sum_{j=1}^{N_{y}} \int_{a}^{b}\left(\mathcal{P r}\left(x, y, t_{n}\right)-r\left(x, y, t_{n}\right)\right)^{+}\left[\mathcal{P}^{-} e_{u}^{n}\right]_{x, j-\frac{1}{2}} d x \\
& -b_{n-1} \int_{a}^{b} \int_{c}^{d}\left(\mathcal{P}^{-} u\left(x, y, t_{0}\right)-u\left(x, y, t_{0}\right)\right) \mathcal{P}^{-} e_{u}^{n} d x d y \\
& \leq\left(\left\|\mathcal{P}^{-} u\left(x, y, t_{n}\right)-u\left(x, y, t_{n}\right)\right\|+\beta\left\|\gamma^{n}(x, y)\right\|\right. \\
& +b_{n-1}\left\|\mathcal{P}^{-} u\left(x, y, t_{0}\right)-u\left(x, y, t_{0}\right)\right\| \\
& \left.+\sum_{i=1}^{n-1}\left(b_{i-1}-b_{i}\right)\left\|\mathcal{P}^{-} u\left(x, y, t_{n-i}\right)-u\left(x, y, t_{n-i}\right)\right\|\right)\left\|\mathcal{P}^{-} e_{u}^{n}\right\| \\
& -\beta \sum_{i=1}^{N_{x}} \int_{c}^{d}\left\{\left(\mathcal{P} q\left(x, y, t_{n}\right)-q\left(x, y, t_{n}\right)\right)^{+}\left[\mathcal{P}^{-} e_{u}^{n}\right]\right. \\
& -\left(\mathcal{P} s\left(x, y, t_{n}\right)-s\left(x, y, t_{n}\right)\right)^{+}\left[\mathcal{P} e_{s}^{n}\right] \\
& \left.-\left(\mathcal{P} p\left(x, y, t_{n}\right)-p\left(x, y, t_{n}\right)\right)^{+}\left[\mathcal{P} e_{p}^{n}\right]\right\}_{i-\frac{1}{2}, y} d y \\
& -\beta \sum_{j=1}^{N_{y}} \int_{a}^{b}\left(\mathcal{P} r\left(x, y, t_{n}\right)-r\left(x, y, t_{n}\right)\right)^{+}\left[\mathcal{P}^{-} e_{u}^{n}\right]_{x, j-\frac{1}{2}} d x \\
& +\left(\sum_{m=1}^{n-1}\left(b_{m-1}-b_{m}\right)\left\|\mathcal{P}^{-} e_{u}^{n-m}\right\|+b_{n-1}\left\|\mathcal{P}^{-} e_{u}^{0}\right\|\right)\left\|\mathcal{P}^{-} e_{u}^{n}\right\| .
\end{aligned}
$$

From the fact that $\varphi \psi \leq \varepsilon \varphi^{2}+\frac{1}{4 \varepsilon} \psi^{2}$, and the property (2.5), we know that 
there exists a positive constant $C$, such that

$$
\begin{aligned}
\int_{a}^{b} \int_{c}^{d}\left(\mathcal{P}^{-} e_{u}^{n}\right)^{2} d x+\frac{\beta}{2} \sum_{i=1}^{N_{x}} \int_{c}^{d}\left(\left[\mathcal{P}^{-} e_{u}^{n}\right]^{2}+\left[\mathcal{P} e_{p}^{n}\right]^{2}+\left[\mathcal{P} e_{s}^{n}\right]^{2}\right)_{i-\frac{1}{2}, y} d y \\
\quad+\beta \tau \sum_{j=1}^{N_{y}} \int_{a}^{b}\left[\mathcal{P}^{-} e_{u}^{n}\right]_{x, j-\frac{1}{2}}^{2} d x \\
\leq C\left(h^{k+1}+(\Delta t)^{2}+(\Delta t)^{\frac{\alpha}{2}} h^{k+\frac{1}{2}}\right)^{2}+\beta \varepsilon \sum_{j=1}^{N_{y}} \int_{a}^{b}\left[\mathcal{P}^{-} e_{u}^{n}\right]_{x, j-\frac{1}{2}}^{2} d x \\
\quad+\frac{\beta}{4} \sum_{i=1}^{N_{x}} \int_{c}^{d}\left(\left[\mathcal{P}^{-} e_{u}^{n}\right]^{2}+\left[\mathcal{P} e_{p}^{n}\right]^{2}+\left[\mathcal{P} e_{s}^{n}\right]^{2}\right)_{i-\frac{1}{2}, y} d y \\
\quad+\left(\sum_{m=1}^{n-1}\left(b_{m-1}-b_{m}\right)\left\|\mathcal{P}^{-} e_{u}^{n-m}\right\|+b_{n-1}\left\|\mathcal{P}^{-} e_{u}^{0}\right\|\right)^{2}+\frac{1}{2} \int_{a}^{b} \int_{c}^{d}\left(\mathcal{P}^{-} e_{u}^{n}\right)^{2} d x
\end{aligned}
$$

Choosing a small enough $\varepsilon \leq \tau$, we can obtain

$$
\begin{aligned}
\left\|\mathcal{P}^{-} e_{u}^{n}\right\|^{2} \leq & C\left(h^{k+1}+(\Delta t)^{2}+(\Delta t)^{\frac{\alpha}{2}} h^{k+\frac{1}{2}}\right)^{2} \\
& +2\left(\sum_{m=1}^{n-1}\left(b_{m-1}-b_{m}\right)\left\|\mathcal{P}^{-} e_{u}^{n-m}\right\|+b_{n-1}\left\|\mathcal{P}^{-} e_{u}^{0}\right\|\right)^{2} .
\end{aligned}
$$

We prove the error estimate by mathematical induction. When $n=1$, the equation (3.10) becomes

$$
\left\|\mathcal{P}^{-} e_{u}^{1}\right\|^{2} \leq C\left(h^{k+1}+(\Delta t)^{2}+(\Delta t)^{\frac{\alpha}{2}} h^{k+\frac{1}{2}}\right)^{2}+2\left\|\mathcal{P}^{-} e_{u}^{0}\right\|^{2} .
$$

It is easy to see that $\left\|\mathcal{P}^{-} e_{u}^{0}\right\| \leq C h^{k+1}$, then

$$
\left\|\mathcal{P}^{-} e_{u}^{1}\right\| \leq C\left(h^{k+1}+(\Delta t)^{2}+(\Delta t)^{\frac{\alpha}{2}} h^{k+\frac{1}{2}}\right) .
$$

Next we suppose the following inequality holds

$$
\left\|\mathcal{P}^{-} e_{u}^{m}\right\| \leq C\left(h^{k+1}+(\Delta t)^{2}+(\Delta t)^{\frac{\alpha}{2}} h^{k+\frac{1}{2}}\right), \quad m=2,3, \ldots, K .
$$

When $n=K+1$, from the equation (3.10), we deduce

$$
\begin{aligned}
\left\|\mathcal{P}^{-} e_{u}^{K+1}\right\|^{2} \leq & C\left(h^{k+1}+(\Delta t)^{2}+(\Delta t)^{\frac{\alpha}{2}} h^{k+\frac{1}{2}}\right)^{2} \\
& +2\left(\sum_{m=1}^{K}\left(b_{m-1}-b_{m}\right)\left\|\mathcal{P}^{-} e_{u}^{K+1-k}\right\|+b_{K}\left\|\mathcal{P}^{-} e_{u}^{0}\right\|\right)^{2} .
\end{aligned}
$$

By the expression (3.2), the assumption (3.11), we can get the following result immediately

$$
\left\|\mathcal{P}^{-} e_{u}^{K+1}\right\| \leq C\left(h^{k+1}+(\Delta t)^{2}+(\Delta t)^{\frac{\alpha}{2}} h^{k+\frac{1}{2}}\right) .
$$

Thus Theorem 2 follows by the triangle inequality and the interpolating property (2.5). 


\section{Conclusion}

In this paper an implicit fully discrete local discontinuous Galerkin (LDG) finite element method is presented for a class of two-dimensional time-fractional Zakharov-Kuznetsov equation. The method is based on a finite difference scheme in time and local discontinuous Galerkin methods in space. Stability is ensured by a careful choice of interface numerical fluxes. We prove that our scheme is unconditional stable and $L^{2}$ error estimate for the linear case with the convergence rate $O\left(h^{k+1}+(\Delta t)^{2}+(\Delta t)^{\frac{\alpha}{2}} h^{k+\frac{1}{2}}\right)$. To date we are not aware of any similar results in published papers. Although not addressed in this paper, the method and analytical technique can also be extended to other kinds of time-fractional equations and higher-dimensional problems easily.

\section{References}

[1] K. Batiha. Approximate analytical solution for the Zakharov-Kuznetsov equations with fully nonlinear dispersion. J. Comput. Appl. Math., 216:157-163, 2008. http://dx.doi.org/10.1016/j.cam.2007.04.020.

[2] H.A. Biagioni and F. Linares. Well-posedness results for the modified ZakharovKuznetsov equation. Progr. Nonlinear Differential Equations Appl., 54:181-189, 2003.

[3] B. Cockburn, G. Kanschat, I. Perugia and D. Schotzau. Superconvergence of the local discontinuous Galerkin method for elliptic problems on Cartesian grids. SIAM J. Numer. Anal., 39:264-285, 2001. http://dx.doi.org/10.1137/S0036142900371544.

[4] B. Cockburn and C.-W. Shu. Tvb Runge-Kutta local projection discontinuous Galerkin finite element method for conservation laws II: general framework. Math. Comp., 52:411-435, 1989. http://dx.doi.org/10.2307/2008474.

[5] W. Deng. Finite element method for the space and time fractional Fokker-Planck equation. SIAM J. Numer. Anal., 47:204-226, 2008. http://dx.doi.org/10.1137/080714130.

[6] A.V. Faminskii. The cauchy problem for the Zakharov-Kuznetsov equation. Differ. Equ., 31:1002-1012, 1995.

[7] G.J. Fix and J.P. Roop. Least squares finite element solution of a fractional order two-point boundary value problem. Comput. Math. Appl., 48:1017-1033, 2004. http://dx.doi.org/10.1016/j.camwa.2004.10.003.

[8] P. LeSaint and P.A. Raviart. On a finite element method for solving the neutron transport equation. In C. de Boor(Ed.), Mathematical Aspects of Finite Elements in Partial Differential Equations. Academic Press, 1974.

[9] Y. Lin and C. Xu. Finite difference/spectral approximations for the timefractional diffusion equation. J. Comput. Phys., 225:1533-1552, 2007. http://dx.doi.org/10.1016/j.jcp.2007.02.001.

[10] F. Liu, P. Zhuang, V. Anh, I. Turner and K. Burrage. Stability and convergence of the difference methods for the space-time fractional advection-diffusion equation. Appl. Math. Comput., 191:12-20, 2007. http://dx.doi.org/10.1016/j.amc.2006.08.162. 
[11] F. Mainardi. Some basic problems in continuum and statistical mechanics. In A. Carpinteri and F. Mainardi(Eds.), Fractals and Fractional Calculus in Continuum Mechanics, Wien, 1997. Springer.

[12] R. Molliq, M.S.M. Noorani, I. Hashim and R.R. Ahmad. Approximate solutions of fractional Zakharov-Kuznetsov equations by VIM. J. Comput. Appl. Math., 233:103-108, 2009. http://dx.doi.org/10.1016/j.cam.2009.03.010.

[13] S. Momani. An explicit and numerical solutions of the fractional KdV equation. Math. Comput. Simulation, 70:110-118, 2005.

http://dx.doi.org/10.1016/j.matcom.2005.05.001.

[14] S. Momani and A. Yildirim. Analytical approximate solutions of the fractional convection-diffusion equation with nonlinear source term by He's homotopy perturbation method. Int. J. Comput. Math., 87:1057-1065, 2010.

http://dx.doi.org/10.1080/00207160903023581.

[15] Z. Odibat and S. Momani. The variational iteration method: An efficient scheme for handling fractional partial differential equations in fluid mechanics. Comput. Math. Appl., 58:2199-2208, 2009.

http://dx.doi.org/10.1016/j.camwa.2009.03.009.

[16] S.A. Sezer, A. Yildirim and S.T. Mohyud-Din. He's homotopy perturbation method for solving the fractional KdV-Burgers-Kuramoto equation. Internat. J. Numer. Methods Heat Fluid Flow, 21:448-458, 2011. http://dx.doi.org/10.1108/09615531111123119.

[17] Y. Xu and C.-W. Shu. Local discontinuous Galerkin methods for two classes of two-dimensional nonlinear wave equations. Phys. D, 208:21-58, 2005. http://dx.doi.org/10.1016/j.physd.2005.06.007.

[18] A. Yildirim and Y. Gülkanat. Analytical approach to fractional ZakharovKuznetsov equations by He's homotopy perturbation method. Commun. Theor. Phys., 53:1005-1010, 2010. http://dx.doi.org/10.1088/0253-6102/53/6/02.

[19] V.E. Zakharov and E.A. Kuznetsov. On three-dimensional solitons. Soviet Phys. JETP, 39:285-286, 1974. 